Pacific Journal of Mathematic 


\title{
STRONG CONTINUITY OF OPERATOR FUNCTIONS
}

\author{
RICHARD V. KADISON
}

\begin{abstract}
The complex-valued functions defined on a subset $S$ of the plane such that $\left(S^{-}-S\right)^{-} \cap S$ is empty which give strongoperator continuous mappings from the set of normal operators on a Hilbert space with spectra in $S$ into the set of all normal operators are characterized as those which are continuous on $S$, bounded on bounded subsets of $S$ and $O(z)$ (Theorem 4.2). In the process of proving this result, it is shown that the adjoint operation is strong-operator continuous on the set of normal operators (Theorem 4.1).
\end{abstract}

In proving his fundamental Density Theorem [1], Kaplansky needs and establishes the fact that continuous real-valued functions vanishing at $\infty$ define strong-operator continuous mappings of the set of bounded self-adjoint operators into itself. He extends this result to bounded continuous functions as well.

While the Kaplansky Density Theorem has become an indispensable tool in the study of operator algebras, the various strong-operator continuity results are themselves important and useful. The purpose of this note is to give a precise delineation of the class of functions which define strong-operator continuous mappings. The technical desirability of having these results for normal operators forces us to consider functions of $n$-tuples of commuting self-adjoint operators (couples would suffice, but $n$-tuples add no difficulties). The results for $n$-tuples appear in $\S 3$; their application to functions of normal operators, in $\S 4$.

The reduction from functions of normal operators to functions of pairs of commuting self-adjoint operators involves the (topological) behavior of the adjoint operation on the normal operators. Now, it is well-known that the the adjoint operation is not strong-operator continuous on the set of all bounded operators. The most familiar example illustrating this discontinuity is the "one-way shift" operator $V$. With $\left\{x_{n}\right\}_{n=1}, 2, \cdots$ an orthonormal basis, $V$ is defined by $V x_{n}=x_{n+1}$, so that $V$ maps the Hilbert space isometrically into itself. The same is true for $V^{m}$, for each positive integer $m$. Thus $\left\|V^{m} x\right\|=1$ for each unit vector $x$ and all positive $m$; so that $\left(V^{m}\right)$ does not tend strongly to 0 . However, if $E_{n}$ is the orthogonal projection with range spanned by $x_{n+1}, x_{n+2}, \cdots, E_{n} V^{n}=V^{n}$. Thus $\left(V^{n}\right)^{*} E_{n}=\left(V^{n}\right)^{*}$; and $\left(V^{n}\right)^{*}$ tends to 0 strongly (since $\left\|\left(V^{n}\right)^{*}\right\|=1$ and $E_{n}$ tends strongly to 0 ). Despite this lack of continuity of the adjoint operation on the set of all bounded operators, it is strong-operator continuous on the 
normal operators. This fact (which seems to be new) is proved in Theorem 4.1.

2. Notation and preliminaries. We deal with complex Hilbert space $\mathscr{C}$. The algebra of all bounded operators on $\mathscr{C}$ is denoted by $\mathscr{B}(\mathscr{L})$. We use the notation $\boldsymbol{R}^{n}$ for real Euclidean $n$-space, and $C$ for the set of complex numbers. The strong-operator topology on $\mathscr{B}(\mathscr{C})$ is the point-open topology on $\mathscr{B}(\mathscr{C})$ induced by the metric topology on $\mathscr{H}$ (so that $\left(A_{n}\right)$ converges to $A$ in the strong-operator topology when $\left(A_{n} x\right)$ converges to $A x$ for each $x$ in $\left.\mathscr{C}\right)$. The strongoperator topology on the Cartesian product $\mathscr{B}(\mathscr{H}) \times \cdots \times \mathscr{B}(\mathscr{H})$ is the product strong-operator topology (with similar terminology for each subset of $\mathscr{B}(\mathscr{C}) \times \cdots \times \mathscr{B}(\mathscr{C}))$.

Definition 2.1. If $A_{1}, \cdots, A_{n}$ is a commuting set of bounded self-adjoint operators on $\mathscr{H}$, the subset $\left\{\left(\rho\left(A_{1}\right), \cdots, \rho\left(A_{n}\right)\right)\right\}$ of $\boldsymbol{R}^{n}$, where $\rho$ ranges through the nonzero multiplicative linear functionals on the $C^{*}$-algebra $\mathfrak{A}$ generated by $A_{1} \cdots, A_{n}$ and $I$ is called the spectrum of $\left(A_{1}, \cdots, A_{n}\right)(=\bar{A})$ and denoted by $\sigma(\bar{A})$. If $S$ is a subset of $\boldsymbol{R}^{n}$, the set of such $\bar{A}$ with $\sigma(\bar{A}) \subseteq S$ is denoted by $\mathscr{B}(\mathscr{H})_{S}$. Since $\mathfrak{A}$ is commutative, it is isomorphic to the algebra of continuous complexvalued functions on some compact Hausdorff space $X$. If $A \rightarrow \hat{A}$ is the isomorphism and $f$ is a real-valued continuous function defined on $S$, we denote by $f\left(A_{1}, \cdots, A_{n}\right)$ the (self-adjoint) operator in $\mathfrak{A}$ corresponding to $x \rightarrow f\left(\hat{A}_{1}(x), \cdots, \hat{A}_{n}(x)\right)$.

In accordance with this definition, $\mathscr{B}(\mathscr{C})_{\boldsymbol{R}}$ will denote the set of all bounded self-adjoint operators on $\mathscr{C}$. We use the notation $\mathscr{B}(\mathscr{C})_{S}$ to denote the set of bounded normal operators on $\mathscr{\mathscr { C }}$ with spectra in $S$, when $S$ is a subset of $C$. Accordingly, $\mathscr{B}(\mathscr{H})_{c}$ will denote the set of all bounded normal operators on $\mathscr{H}$. With $f$ a continuous real-valued function defined on a subset $S$ of $R^{n}$, we use the symbol $f$, again, to denote the mapping of $\mathscr{B}(\mathscr{C})_{S}$ into $\mathscr{B}(\mathscr{C})_{\boldsymbol{R}}$ described in Definition 2.1. By means of Spectral Theory, we can ascribe a meaning to $f\left(A_{1}, \cdots, A_{n}\right)$ for certain noncontinuous functions $f$ on $S$.

For a point $x=\left(x_{1}, \cdots, x_{n}\right)$ in $\boldsymbol{R}^{n}$, we denote by $|x|$ the sum $\left|x_{1}\right|+\cdots+\left|x_{n}\right|$ and by $\|x\|$ the number $\left(\sum x_{j}^{2}\right)^{1 / 2}$. We use the notation " $f$ is $O(x)$ ", for a function $f$ defined on a subset $S$ of $\boldsymbol{R}^{n}$, to mean $x \rightarrow f(x) /\|x\|$ is bounded on $S$ outside some bounded subset of $S$.

3. Operator functions of several variables. We determine conditions, in this section, for real-valued functions defined on certain subsets $S$ of $\boldsymbol{R}^{n}$ to be strong-operator continuous on $\mathscr{B}(\mathscr{C})_{S}$. Basic to this discussion is the: 
REMARK 3.1. The mapping $\left(A_{1}, \cdots, A_{n}\right) \rightarrow A_{1} \cdots A_{n}$ is strongoperator continuous on bounded subsets of $\mathscr{B}(\mathscr{C}) \times \cdots \times \mathscr{B}(\mathscr{H})$.

Lemma 3.2. If $f$ is a continuous mapping of $\boldsymbol{R}^{n}$ into $\boldsymbol{R}$ which tends to a limit at $\infty$ then $f$ is strong-operator continuous on $\mathscr{B}(\mathscr{H})_{R} n$.

Proof. Let $X$ be the one-point compactification of $\boldsymbol{R}^{n}$; and let $\mathscr{A}$ be the algebra of finite linear combinations of products $f_{1} \cdots f_{n}$ where $f_{j}$ is a continuous real-valued function on $\boldsymbol{R}$ of bound not exceeding 1 and tending to a limit at $\infty$. The constant function 1 is in $\mathscr{A}$. If $a$ and $b$ are distinct points of $X$ and both lie in $\boldsymbol{R}^{n}$, suppose $a$ and $b$ have distinct $j$ th coordinates $a_{j}, b_{j}$. We can construct $f_{j}$ on $\boldsymbol{R}$ such that $\left\|f_{j}\right\|=1, f_{j}\left(a_{j}\right)=1$ and $f_{j}$ vanishes outside an open interval about $a_{j}$ not containing $b_{j}$. Choosing $f_{k}$ to be 1 for $k \neq j$, $f_{1} \cdots f_{n}$ is in $\mathscr{A}$ has the value 1 at $\alpha$ and 0 at b. If $a$ is in $\boldsymbol{R}^{n}$, say $a=\left(a_{1}, \cdots, a_{n}\right)$, construct $f_{j}$ on $\boldsymbol{R}$ with $f_{j}\left(a_{j}\right)=1=\left\|f_{j}\right\|$ and $f_{j}$ vanishing at $\infty$. Then $f_{1} \cdots f_{n}$ is 1 at $a$ and 0 at $\infty$. Thus $\mathscr{A}$ contains the constants and separates points of $X$. From the StoneWeierstrass Theorem, $\mathscr{A}$ is uniformly dense in $C(X)$.

If we have established the strong-operator continuity of each function in $\mathscr{A}$ on $\mathscr{B}(\mathscr{C}) \boldsymbol{R}^{n}$, then that of $f$ will follow. In fact, given $A_{1}, \cdots, A_{n}$ commuting self-adjoint operators and $x$ in $\mathscr{C}$, select vectors $y_{k}^{(j)}, k=1, \cdots, m ; j=1, \cdots, n$ in $\mathscr{H}$ such that if $\left\|\left[A_{j}-B_{j}\right] y_{k}^{(j)}\right\|<1, k=1, \cdots, m ; j=1, \cdots, n$, with $B_{1}, \cdots, B_{n}$ commuting self-adjoint operators, then

$$
\left\|\left[g\left(A_{1}, \cdots, A_{n}\right)-g\left(B_{1}, \cdots, B_{n}\right)\right] x\right\|<1 / 3,
$$

where $g$ is a function in $\mathscr{A}$ such that $\|f-g\|<1 / 3\|x\|$. For this $\left(B_{1}, \cdots, B_{n}\right)$,

$$
\begin{aligned}
& \left\|\left[f\left(A_{1}, \cdots, A_{n}\right)-f\left(B_{1}, \cdots, B_{n}\right)\right] x\right\| \\
& \leqq \\
& \quad\left\|\left[f\left(A_{1}, \cdots, A_{n}\right)-g\left(A_{1}, \cdots, A_{n}\right)\right] x\right\| \\
& \quad+\left\|\left[g\left(A_{1}, \cdots, A_{n}\right)-g\left(B_{1}, \cdots, B_{n}\right)\right] x\right\| \\
& \quad+\left\|\left[g\left(B_{1}, \cdots, B_{n}\right)-f\left(B_{1}, \cdots, B_{n}\right)\right] x\right\|<1 .
\end{aligned}
$$

The continuity of $g$ in $\mathscr{A}$ will follow from that of the products $f_{1} \cdots f_{n}$ used in the definition of $\mathscr{A}$. Since each $f_{j}$ is strong-operator continuous on $\mathscr{B}(\mathscr{C})_{\boldsymbol{R}}\left[1\right.$; Lemma 5] and $\left(A_{1}, \cdots, A_{n}\right) \rightarrow A_{1} \cdots A_{n}$ is strong-operator continuous on $\mathscr{B}(\mathscr{C})_{1} \times \cdots \times \mathscr{B}(\mathscr{C})_{1}$, where $\mathscr{B}(\mathscr{C})_{1}$ is the unit ball in $\mathscr{B}(\mathscr{C})$, the composite mapping

$$
\begin{aligned}
& \left(B_{1}, \cdots, B_{n}\right) \rightarrow\left(f_{1}\left(B_{1}\right), \cdots, f_{n}\left(B_{n}\right)\right) \rightarrow f_{1}\left(B_{1}\right) \cdots f_{n}\left(B_{n}\right) \\
& \quad=\left(f_{1} \cdots f_{n}\right)\left(B_{1}, \cdots, B_{n}\right)
\end{aligned}
$$


(recall that $\left\|f_{j}\right\| \leqq 1$ so that $\left\|f_{j}\left(B_{j}\right)\right\| \leqq 1$, and compare Remark 3.1) is continuous.

With $f$ a real-valued function defined on a subset $S$ of $\boldsymbol{R}^{n}$, a jump point for $f$ is a point in $S^{-}$for which $\left(\lim _{f} f\right)(p)-(\lim f)(p)>0$. If $f$ is continuous, the jump points for $f$ lie in $S^{-}-\bar{S}$. We shall need the following lemma whose proof is a slight variation of the proof of $[1 ;$ Th. 2] to suit the present circumstances.

LEMмA 3.3. If $h$ is a bounded, real-valued function on the subset $S$ of $\boldsymbol{R}^{n}$ and the set $J$ of jump points for $h$ is such that $J^{-} \cap S$ is null, then $h$ is strong-operator continuous on $\mathscr{B}(\mathscr{C})_{s}$.

Proof. Suppose $\left(A_{1}, \cdots, A_{m}\right)(=\bar{A})$ is in $\mathscr{B}(\mathscr{C})_{S}$. Then $\sigma(\bar{A})$ is a compact subset of $\boldsymbol{R}^{n}$ disjoint from $J^{-}$(by assumption). Let $O$ be a bounded open set containing $\sigma(\bar{A})$ with closure $O^{-}$disjoint from $J^{-}$. Since no jump point for $h$ lies in $O^{-} \cap S^{-}$, assigning $(\overline{\lim } h)(p)$ to each $p$ of this set defines a continuous extension of $h$ to it. Finally, let $h_{0}$ be the function on $\boldsymbol{R}^{n}$ which is some continuous extension $h_{1}$ to $O^{-}$of this function (Tietze Extension Theorem), $h$ on $S$ and 0 elsewhere. We note that, with $k$ continuous on $\boldsymbol{R}^{n}, 1$ on $\sigma(\bar{A})$ and 0 outside $O, h_{0} k(=p)$ and $1-k+h_{0} k(=q)$ are continuous on $\boldsymbol{R}^{n}$. On the complement of $\mathrm{O}^{-}, k$ and hence $p$ are 0 ; so that $p$ is continuous at points of this complement (an open set). On $O^{-}-O, k$ is 0 ; so that $p$ is 0 and continuous at points of $O^{-}-O$, since $p=h_{1} k$ on $O^{-}$ with $h_{1}$ continuous, hence bounded, on $\mathrm{O}^{-}$. On $O$, an open set, $p$ is the product of the two continuous functions $h_{1}$ and $k$. Since $p$ and $q-1$ vanish outside $O$ and are continuous on $\boldsymbol{R}^{n}$, they are strongoperator continuous on $\mathscr{C}(\mathscr{C}) \boldsymbol{R}^{n}$ (from Lemma 3.2).

As $p=q=h$ on $\sigma(\bar{A}), p(\bar{A})=q(\bar{A})=h(\bar{A})$. Combining this with the identity $h_{0}=\left(1-h_{0}\right) p+h_{0} q$ which becomes $h=(1-h) p+h q$ on $S$; we have, for each $\bar{B}$ in $\mathscr{B}(\mathscr{H})_{S}$,

$$
h(\bar{B})-h(\bar{A})=[1-h(\bar{B})][p(\bar{B})-p(\bar{A})]+h(\bar{B})[q(\bar{B})-q(\bar{A})] .
$$

The strong-operator continuity of $h$ on $\mathscr{B}(\mathscr{C})_{S}$ follows from that of $p$ and $q$, this last identity and the fact that $h$ is bounded on $S$.

THEOREM 3.4. If $f$ is a real-valued function defined and $O(x)$ on a subset $S$ of $\boldsymbol{R}^{n}$, bounded on bounded subsets of $S$ and such that $J^{-} \cap S$ is null, where $J$ is the set of jump points of $f$, then $f$ is strong-operator continuous on $\mathscr{B}(\mathscr{C})_{S}$.

Proof. We note, first, that if $g$ is bounded, with jump points in $J$, and real-valued on $S$, and $h$ is strong-operator continuous on 
$\mathscr{B}(\mathscr{C})_{S}$, then $g h$ is strong-operator continuous on $\mathscr{B}(\mathscr{C})_{S}$. This follows from the strong-operator continuity of $h$, of $g$ (from Lemma 3.3), and the inequality:

$$
\begin{aligned}
& \|[g(\bar{A}) h(\bar{A})-g(\bar{B}) h(\bar{B})] x\| \\
& \quad \leqq\|g(\bar{A})\| \cdot\|[h(\bar{A})-h(\bar{B})] x\|+\|[g(\bar{A})-g(\bar{B})] h(\bar{B}) x\|,
\end{aligned}
$$

where $\bar{A}=\left(A_{1}, \cdots, A_{n}\right)$ and $\bar{B}=\left(B_{1}, \cdots, B_{n}\right)$ are in $\mathscr{B}(\mathscr{C})_{S}$.

Let $g(x)$ be $f(x) /(1+|x|)$ for $x$ in $S$,

$$
\begin{aligned}
x=\left(x_{1}, \cdots, x_{n}\right),|x|=\left|x_{1}\right|+\cdots+\left|x_{n}\right| & (\geqq\|x\| \\
& \left.=\left(\sum\left|x_{j}\right|^{2}\right)^{1 / 2}\right) .
\end{aligned}
$$

From the hypothesis, $g$ is bounded on $S$; and its set of jump points is contained in $J$. Once we note that $x \rightarrow|x|$ is strong-operator continuous on $\mathscr{B}(\mathscr{C})_{S}$, the strong-operator continuity of $g$ on $\mathscr{B}(\mathscr{H})_{S}$ (Lemma 3.3) and the argument of the first paragraph gives the strongoperator continuity on $\mathscr{B}(\mathscr{C})_{S}$ of $h$ defined by $h(x)=(1+|x|) g(x)$, for $x$ in $S$. Since $\left|\left(A_{1}, \cdots, A_{n}\right)\right|=\left|A_{1}\right|+\cdots+\left|A_{n}\right|$, the strongoperator continuity of $x \rightarrow|x|$ on $\mathscr{B}(\mathscr{C}) \boldsymbol{R}^{n}$ will follow from that of $A \rightarrow|A|$ on $\mathscr{B}(\mathscr{C})_{R}$. Let $r(x)$ be $x$ for $|x| \leqq 1$ and $|x| / x$ for $1 \leqq|x|$; $s(x)$ be $x r(x)$; and $t(x)$ be $|x|-s(x)$. Since $r$ is bounded, $t$ vanishes outside $[-1,1]$ and both are continuous on $R$, [1; Th. 2, Lemma 5] shows that both are strong-operator continuous on $\mathscr{B}(\mathscr{C})_{R}$. So is $s$, from the argument of the first paragraph. Thus, $x \rightarrow|x|=s(x)+t(x)$ is strong-operator continuous on $\mathscr{B}(\mathscr{C})_{R}$.

Our thanks are due to R. J. Blattner for suggesting ' $1+|x|$ ' in place of ' $|x|$ ' to define $g$ thereby correcting and simplifying the argument.

LEMmA 3.5. With $S$ a subset of $\boldsymbol{R}^{n}$, if the real-valued function $f$ is strong-operator continuous on $\mathscr{B}(\mathscr{C})_{S}$ it is continuous on $S$, bounded on bounded subsets of $S$, and $O(x)$.

Proof. Assuming $f$ is defined on $\mathscr{B}(\mathscr{C})_{S}$ (by Spectral Theory) and restricting $f$ to $\left\{\left(a_{1} I, \cdots, a_{n} I\right):\left(a_{1}, \cdots, a_{n}\right)\right.$ in $\left.S\right\}$, we see that $f$ must be continuous on $S$ if it is to be strong-operator continuous on $\mathscr{B}(\mathscr{C})_{S}$. With $x_{0}$ in $S$, the translated set, $S-x_{0}$, contains 0 ; and $g$ defined on $S-x_{0}$ by $g(x)=f\left(x+x_{0}\right)-f\left(x_{0}\right)$ is bounded on bounded subsets of $S-x_{0}$ and $O(x)$ if and only if $f$ is bounded on bounded subsets of $S$ and $O(x)$. We may assume that 0 lies in $S$ and $f(0)$ is 0 .

Suppose that $f$ is not $O(x)$. Then there is a sequence $\left(x_{m}\right)$ in $S$ with $\left\|x_{m}\right\| \rightarrow \infty$ such that $m\left\|x_{m}\right\| \leqq\left|f\left(x_{m}\right)\right|$. Taking $\mathscr{L}_{2}(0,1)$ for $\mathscr{H}$ (relative to Lebesgue measure), we show that $f$ is not strongoperator continuous at $(0, \cdots, 0)$ on $n$-tuples of multiples (by coordi- 
nates of the $x_{m}$ 's) of a projection in the multiplication algebra of $\mathscr{L}_{2}(0,1)$. More specifically, given $\psi_{1}^{(j)}, \cdots, \psi_{m}^{(j)}, j=1, \cdots, n$, in $\mathscr{L}_{2}(0,1)$, we find a subset $X$ of $(0,1)$ having positive measure and $r$ such that, with $g_{j}=a_{j}$ on $X$ and 0 on the complement of $X$, where $x_{r}=\left(a_{1}, \cdots, a_{n}\right), \int\left|g_{j} \psi_{p}^{(j)}\right|^{2} \leqq 1$ for $j=1, \cdots, n ; p=1, \cdots, m$; while $\int|f \circ g|^{2} \geqq 1$, where $g(s)=\left(g_{1}(s), \cdots, g_{n}(s)\right)$ for $s$ in $(0,1)$. With $M_{g_{j}}$ the multiplication operator (on $\mathscr{L}_{2}(0,1)$ ) corresponding to $g_{j}$, $\left(M_{g_{1}}, \cdots, M_{g_{n}}\right) \in \mathscr{\mathscr { S }}(\mathscr{C})_{S}$ and $f\left(M_{g_{1}}, \cdots, M_{g_{n}}\right)=M_{f \circ g}$. Thus

$$
\left\|f\left(M_{g_{1}}, \cdots, M_{g_{n}}\right)(1)\right\| \geqq 1
$$

despite the fact that $\left\|M_{g_{j}} \psi_{p}^{(j)}\right\| \leqq 1$ for $p=1, \cdots, m ; j=1, \cdots, n$. Hence $f$ is not strong-operator continuous on $\mathscr{B}(\mathscr{C})_{S}$.

It remains to locate $X$ and $r$ as described. With $\psi=\sum_{j, p}\left|\psi_{p}^{(j)}\right|$, let $X_{k}$ be the subset of $(0,1)$ at which $|\psi|$ does not exceed $k$, for $k=1,2, \cdots$. Since $\psi$ is in $\mathscr{L}_{2}(0,1), X_{k}$ has positive measure $a$ for some $k$. Choose $r$ larger than $k$ so that $\left\|x_{r}\right\|^{2} a k^{2} \geqq 1$; and let $b$ be $\left(\left\|x_{r}\right\|^{2} a k^{2}\right)^{-1}$. Then $0<b \leqq 1$, and there is a subset $X$ of $X_{k}$ with measure $a b$. Defining $g_{j}$ to be $a_{j}$ at points of $X$ and 0 at points of the complement of $X$, where $x_{r}=\left(a_{1}, \cdots, a_{n}\right)$, we have

$$
\int\left|g_{j} \psi\right|^{2}=\int_{X}\left|g_{j} \psi\right|^{2} \leqq k^{2} \int\left|g_{j}\right|^{2}=k^{2}\left|a_{j}\right|^{2} a b \leqq k^{2}\left\|x_{r}\right\|^{2} a b=1,
$$

while

$$
\int|f \circ g|^{2} \geqq \int_{X}|f \circ g|^{2}=f\left(x_{r}\right)^{2} a b \geqq r^{2}\left\|x_{r}\right\|^{2} a b=r^{2} k^{-2} \geqq 1 .
$$

Since

$$
\sum_{p=1}^{m} \int\left|g_{j} \psi_{p}^{(j)}\right|^{2} \leqq \int\left|g_{j} \psi\right|^{2} \leqq 1
$$

we have $\int\left|g_{j} \psi_{p}^{(j)}\right|^{2} \leqq 1$, for $p=1, \cdots, m$ and $j=1, \cdots, n$.

Suppose, next, that $f$ is not bounded on some bounded subset of $S$. Then there is a sequence $\left(x_{m}\right)$, with $x_{m}$ in $S$, tending to some point $x_{0}$ in $\boldsymbol{R}^{n}$ such that $m \leqq\left|f\left(x_{m}\right)\right|$. As before, translating by $-x_{0}$, we may assume that $x_{0}=0$. Select $\left(b_{1}, \cdots, b_{n}\right)$ in $S$ with $\left|b_{j}\right| \leqq 1$, $j=1, \cdots, n$.

We shall show that $f$ is not strong-operator continuous at $\left(b_{1} I, \cdots, b_{n} I\right)$ on $\mathscr{B}(\mathscr{C})_{s}$. Given $\psi_{k}^{(j)}, j=1, \cdots, n ; k=1, \cdots, m$ in $\mathscr{L}_{2}(0,1)$; let $\psi=\sum_{p, k}\left|\psi_{k}^{(p)}\right|$. There is a subset $X$ of $(0,1)$ with positive Lebesgue measure $a$ such that $\int_{X}|\psi|^{2} \leqq 1 / 4$. Choose $r$ so that $\left|a_{j}\right| \leqq 1, j=1, \cdots, n$, where $x_{r}=\left(a_{1}, \cdots, a_{n}\right)$; and so that $a\left|f\left(x_{r}\right)-f\left(b_{1}, \cdots, b_{n}\right)\right|^{2} \geqq 1$. Let 
$g_{j}$ be $a_{j}$ on $X$ and $b_{j}$ on the complement of $X$ in $(0,1)$.

As before, $f\left(M_{g_{1}}, \cdots, M_{g_{n}}\right)=M_{f \circ g}$, where $g(s)=\left(g_{1}(s), \cdots, g_{n}(s)\right)$ for $s$ in $(0,1)$. Thus $\|\left[f\left(M_{g_{1}}, \cdots, M_{g_{n}}\right)-f\left(b_{1} I, \cdots, b_{n} I\right] 1 \| \geqq 1\right.$, since

$$
\begin{aligned}
\int \mid f & \circ g-\left.f\left(b_{1}, \cdots, b_{n}\right)\right|^{2} \\
\quad & =\int_{X}\left|f\left(x_{r}\right)-f\left(b_{1}, \cdots, b_{n}\right)\right|^{2}=a\left|f\left(x_{r}\right)-f\left(b_{1}, \cdots, b_{n}\right)\right|^{2} \geqq 1 .
\end{aligned}
$$

But

$$
\begin{aligned}
& \int\left|\left(b_{j}-g_{j}\right) \psi_{k}^{(j)}\right|^{2} \leqq \int\left|b_{j}-g_{j}\right|^{2}\left(\sum_{p, k}\left|\psi_{k}^{(p)}\right|\right)^{2} \\
& \quad=\int\left|\left(b_{j}-g_{j}\right) \psi\right|^{2}=\int_{X}\left|\left(b_{j}-a_{j}\right) \psi\right|^{2} \leqq 4 \int_{X}|\psi|^{2} \leqq 1,
\end{aligned}
$$

so that $\left\|\left(b_{j} I-M_{g_{j}}\right) \psi_{k}^{(j)}\right\| \leqq 1$, for $j=1, \cdots, n$ and $k=1, \cdots, m$. As $\left(M_{g_{1}}, \cdots, M_{g_{n}}\right) \in \mathscr{B}(\mathscr{C})_{s}, f$ is not strong-operator continuous at $\left(b_{1} I, \cdots, b_{n} I\right)$ on $\mathscr{R}(\mathscr{C})_{s}$, completing the proof of this lemma.

Combining Theorem 3.4 with the foregoing lemma, we have:

THEOREM 3.6. If $S$ is a subset of $\boldsymbol{R}^{n}$ such that $\left(S^{-}-S\right)^{-} \cap S$ is empty then a real-valued function $f$ defined on $S$ is strong-operator continuous on $\mathscr{B}(\mathscr{C})_{S}$ if and only if it is continuous on $S$, bounded on bounded subsets of $S$, and $O(x)$.

Proof. In view of Theorem 3.4 and Lemma 3.5, we need note only that the set of jump points of a function continuous on $S$ is a subset of $S^{-}-S$.

For a closed set $S, S^{-}-S$ is empty; and, for an open set $S, S^{-}-S$ is closed. In both cases $\left(S^{-}-S\right)^{-} \cap S$ is empty; from which we have:

CoRollary 3.7. If $S$ is a closed or open subset of $\boldsymbol{R}^{n}$, a realvalued function defined on $S$ is strong-operator continuous on $\mathscr{B}(\mathscr{H})_{S}$ if and only if it is continuous on $S$, bounded on bounded subsets of $S$, and $O(x)$.

Of course, the continuity assumption makes the hypothesis of boundedness on bounded subsets superfluous when $S$ is a closed set.

4. Functions of normal operators. The key to applying the results of $\S 3$ to the normal operators $\mathscr{B}(\mathscr{C})_{c}$ is:

THEOREM 4.1. The adjoint operation is strong-operator continuous on $\mathscr{B}(\mathscr{C})_{C}$. 
Proof. The assertion follows from:

$$
\begin{aligned}
& \left\|\left(B^{*}-A^{*}\right) x\right\|^{2}=\|B x\|^{2}-\|A x\|^{2}+\left(x,(A-B) A^{*} x\right) \\
& \quad+\left((A-B) A^{*} x, x\right) \leqq\|(A-B) x\|(\|A x\|+\|B x\|) \\
& \quad+2 \| A-B) A^{*} x\|\| x \| .
\end{aligned}
$$

(Our original proof of Theorem 4.1 was somewhat longer. A. Hoppenwasser found a simpler proof which led us to the argument above.)

THEOREM 4.2. With $f$ a complex-valued function defined on a subset $S$ of $C$ for which $\left(S^{-}-S\right)^{-} \cap S$ is empty (in particular, for $S$ open or closed), $f$ is strong-operator continuous on $\mathscr{B}(\mathscr{H})_{S}$ if and only if $f$ is continuous, bounded on bounded subsets of $S$, and $O(z)$.

Proof. Adopting the usual identification of $\boldsymbol{C}$ with $\boldsymbol{R}^{2}$, we may view $S$ as a subset of $\boldsymbol{R}^{2}$. With $z=a+i b, a$ and $b$ real, let $f(z)=$ $g(a, b)+i h(a, b), g(a, b)$ and $h(a, b)$ real. Then $g$ and $h$ are defined on $S$. Moreover, $g$ and $h$ are continuous on $S$, bounded on bounded subsets of $S$, and $O(z)$, if and only if the same are true for $f$. This is the case if and only if $g$ and $h$ are strong-operator continuous on $\mathscr{B}(\mathscr{H})_{S}$, from Theorem 3.6.

We conclude the proof by showing that

$$
A_{1}+i A_{2} \rightarrow g\left(A_{1}, A_{2}\right)+i h\left(A_{1}, A_{2}\right)=f\left(A_{1}+i A_{2}\right)
$$

is strong-operator continuous if and only if $g$ and $h$ are. Since

$$
\begin{aligned}
A_{1}+i A_{2} & \rightarrow\left(\frac{1}{2}\left[A_{1}+i A_{2}+\left(A_{1}+i A_{2}\right)^{*}\right],\right. \\
& \left.\frac{1}{2 i}\left[A_{1}+i A_{2}-\left(A_{1}+i A_{2}\right)^{*}\right]\right)=\left(A_{1}, A_{2}\right)
\end{aligned}
$$

is a strong-operator homeomorphism of $\mathscr{B}(\mathscr{H})_{\boldsymbol{C}}$ with $\mathscr{B}(\mathscr{C}) \boldsymbol{R}^{2}$, from Theorem 4.1, it will suffice to show that $\left(A_{1}, A_{2}\right) \rightarrow g\left(A_{1}, A_{2}\right)+i h\left(A_{1}, A_{2}\right)$ is strong-operator continuous if and only if $g$ and $h$ are. All that requires proof is the strong-operator continuity of $g$ and $h$ on $\mathscr{B}(\mathscr{C})_{S}$ from that of $\left(A_{1}, A_{2}\right) \rightarrow g\left(A_{1}, A_{2}\right)+i h\left(A_{1}, A_{2}\right)$ on $\mathscr{B}(\mathscr{C})_{S}$. From Theorem 4.1, $\left(A_{1}, A_{2}\right) \rightarrow\left[g\left(A_{1}, A_{2}\right)+i h\left(A_{1}, A_{2}\right)+\left(g\left(A_{1}, A_{2}\right)+i h\left(A_{1}, A_{2}\right)\right)^{*}\right] / 2=g\left(A_{1}, A_{2}\right)$ is strong-operator continuous on $\mathscr{B}(\mathscr{\mathscr { C }})_{S}$, and similarly for $\left(A_{1}, A_{2}\right) \rightarrow$ $h\left(A_{1}, A_{2}\right)$.

We have made no distinction between $\mathscr{B}(\mathscr{C})_{S}$ with $S$ a subset of $C$, referring to the normal operators on $\mathscr{C}$ with spectra in $S$, and $\mathscr{B}(\mathscr{C})_{S}$ with $S$ a subset of $\boldsymbol{R}^{2}$, referring to pairs of commuting selfadjoint operator with joint spectrum in $S$. The context makes clear 
the sense in which this notation applies; and the argument indicates that there is no essential distinction between the sets designated. Of course, a theorem analogous to Theorem 3.4 holds for functions of normal operators.

\section{REFERENCE}

1. I. Kaplansky, A theorem on rings of operators, Pacific J. Math. 1 (1951) 227-232. Received May 26, 1967. Research supported in part by ONR and NSF.

University of Pennsylvania 



\section{PACIFIC JOURNAL OF MATHEMATICS}

\section{EDITORS}

\author{
H. ROYDEN \\ Stanford University \\ Stanford, California \\ R. R. Phelps \\ University of Washington \\ Seattle, Washington 98105
}

J. DUGUNDJI

Department of Mathematics

University of Southern California

Los Angeles, California 90007

RICHARD ARENS

University of California

Los Angeles, California 90024

\section{ASSOCIATE EDITORS}
E. F. BECKENBACH
B. H. NeumanN
F. WOLF
K. YosidA

\section{SUPPORTING INSTITUTIONS}

UNIVERSITY OF BRITISH COLUMBIA

CALIFORNIA INSTITUTE OF TECHNOLOGY

UNIVERSITY OF CALIFORNIA

MONTANA STATE UNIVERSITY

UNIVERSITY OF NEVADA

NEW MEXICO STATE UNIVERSITY

OREGON STATE UNIVERSITY

UNIVERSITY OF OREGON

OSAKA UNIVERSITY

UNIVERSITY OF SOUTHERN CALIFORNIA
STANFORD UNIVERSITY

UNIVERSITY OF TOKYO

UNIVERSITY OF UTAH

WASHINGTON STATE UNIVERSITY

UNIVERSITY OF WASHINGTON

AMERICAN MATHEMATICAL SOCIETY CHEVRON RESEARCH CORPORATION TRW SYSTEMS

NAVAL WEAPONS CENTER

Printed in Japan by International Academic Printing Co., Ltd., Tokyo, Japan 


\section{Pacific Journal of Mathematics}

\section{Vol. 26, No. $1 \quad$ November, 1968}

Efraim Pacillas Armendariz, Closure properties in radical theory......... 1

Friedrich-Wilhelm Bauer, Postnikov-decompositions of functors .......... 9

Thomas $\mathrm{Ru}-$ Wen Chow, The equivalence of group invariant positive definite

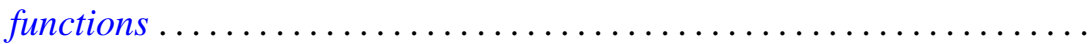

Thomas Allan Cootz, A maximum principle and geometric properties of

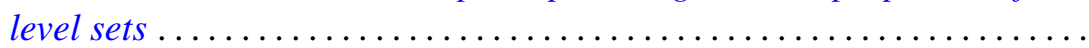

Rodolfo DeSapio, Almost diffeomorphisms of manifolds ............ 47

R. L. Duncan, Some continuity properties of the Schnirelmann density......

Ralph Jasper Faudree, Jr., Automorphism groups of finite subgroups of

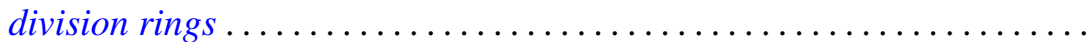

Thomas Alastair Gillespie, An invariant subspace theorem of $J$.

Feldman.........................................

George Isaac Glauberman and John Griggs Thompson, Weakly closed direct factors of Sylow subgroups .............................

Hiroshi Haruki, On inequalities generalizing a Pythagorean functional equation and Jensen's functional equation .....................

David Wilson Henderson, D-dimension. I. A new transfinite dimension.....

David Wilson Henderson, D-dimension. II. Separable spaces and

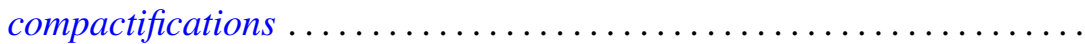

Julien O. Hennefeld, A note on the Arens products ............... 115

Richard Vincent Kadison, Strong continuity of operator functions ...

J. G. Kalbfleisch and Ralph Gordon Stanton, Maximal and minimal coverings of $(k-1)$-tuples by $k$-tuples.

Franklin Lowenthal, On generating subgroups of the Moebius group by pairs of infinitesimal transformations...

Michael Barry Marcus, Gaussian processes with stationary increments possessing discontinuous sample paths . .

Zalman Rubinstein, On a problem of Ilyeff ...

Bernard Russo, Unimodular contractions in Hilbert space. ...

David Lee Skoug, Generalized Ilstow and Feynman integrals...

William Charles Waterhouse, Dual groups of vector spaces . 